\title{
On the Ethics of Social
}

\section{Network Research in Libraries}

\section{Authors: Sara Mannheimer, Scott W.H. Young, Doralyn Rossmann}

This is a post-print of an article in volume 14, issue 2 of the Journal of Information,

Communication, and Ethics in Society. http://www.emeraldinsight.com/journal/jices

Date uploaded: 02 January 2016

Suggested Citation: Mannheimer S, Young SWH, Rossmann D (2016) On the Ethics of Social Network Research in Libraries. Journal of Information, Communication, and Ethics in Society 14(2). http://doi.org/10.1108/JICES-05-2015-0013 


\title{
On The Ethics of Social Network Research in Libraries
}

\author{
Sara Mannheimer \\ Montana State University \\ Bozeman, MT, USA \\ sara.mannheimer@montana.edu
}

\author{
Scott W. H. Young \\ Montana State University \\ Bozeman, MT, USA \\ swyoung@montana.edu
}

\author{
Doralyn Rossmann \\ Montana State University \\ Bozeman, MT, USA \\ doralyn@montana.edu
}

\begin{abstract}
Purpose

In this paper, faculty librarians at Montana State University explore the ethical dimensions of conducting research with user-generated social networking site (SNS) data. In an effort to guide Librarian-Researchers, this paper first offers a background discussion of privacy ethics across disciplines, then proposes a library-specific ethical framework for conducting SNS research. Design

By surveying the literature in other disciplines, three key considerations are identified that can inform ethical practice in the field of Library Science: context, expectation, and value analysis. For each of these considerations, the framework is tailored to consider ethical issues as they relate to libraries and our practice as Librarian-Researchers.
\end{abstract}

\section{Findings}

The unique role of the Librarian-Researcher demands an ethical framework specific to that practice. The findings of this paper propose such a framework.

\section{Practical Implications}

Librarian-Researchers are at a unique point in our history. In exploring SNSs as a source of data to conduct research and improve services, we become challenged by conflicting and equally cherished values of patron privacy and information access. By evaluating research according to context, expectations, and value, this framework provides an ethical path forward for research using SNS data.

\section{Originality/Value}

As of this article's publication, there is no existing ethical framework for conducting SNS research in libraries. The proposed framework is informed both by library values and by broader research values, and therefore provides unique guidelines for the Librarian-Researcher.

\section{INTRODUCTION}

The growing practice of collecting and analyzing user-generated data from Social Networking Services (SNSs) has given rise to new ethical questions and considerations. As librarians conducting SNS research, we must additionally contend with long-standing professional values that protect patron privacy. We explore these questions through a review of ethical practice in SNS research in other disciplines, and then by drawing parallels to the library profession. A case study description of SNS-based research conducted by academic librarians at Montana State 
University (MSU) illustrates some of the challenges inherent in SNS research in libraries. This article proposes a loosening of a traditional absolutist view of patron privacy, and suggests an ethical framework for SNS-based research in libraries centered around context, expectations, and value analysis.

\section{LITERATURE REVIEW}

\section{SNS Ethics Discussion Across Disciplines}

SNSs provide communication infrastructure and exchange on a potentially massive scale. When SNS users discuss their lives, publish items of interest, and interact with their communities, these activities are documented through the SNS, and can be collected into large-scale datasets that are often made available by SNSs via machine access tools such as APIs. Consequently, SNS data collection and analysis represent new opportunities for research across disciplines. Researchers have used SNSs to measure public opinion (Jansen et al., 2009; Tumasjan et al., 2010), to analyze online communities (Beaudoin and Tao, 2007), and as a pedagogical tool (Rinaldo et al., 2011), and the research applications for SNSs continue to grow. As the possibilities for SNS research have developed, so have questions of privacy and ethics with regards to user data generated through SNSs. These questions have been especially prevalent throughout the literature of computer science (Obole and Welsh, 2012; Elovici et al., 2013), social sciences (Wilkinson and Thelwall, 2011; Breiger, 2005), environmental science (Soranno et al., 2014), and the medical sciences (Gustafson and Woodworth, 2014; Kaye, 2015). Ethical and normative assumptions have been challenged within these disciplines, with many researchers noting a lack of ethical limits, standards, or protocols for research involving SNS user data (boyd and Ellison, 2007; Burgess Bruns, 2012; Henderson et al., 2012; Alim, 2014). Anonymity, confidentiality, comfort, and consent of research subjects emerge as thematic issues. Neuhaus and Webmoor (2011) argue that existing ethical protocols are not adequate for SNS research, as SNS datasets represent an unprecedented level of scale and personalization: "With large data sets, the individual disappears in the mass, but the issue and potential risk remain on the scale of the individual" (p. 58). In response, many studies propose new frameworks for ethically conducting research with SNS user data (Hutton and Henderson, 2013; van Wynsberghe et al., 2013; Langheinrich et al., 2013; Datig and Russell, 2014; Rivers and Lewis, 2014). The summation of these studies is twofold: context and expectation. Addressing context, van Wynsberghe, Been, and van Keulen write, "Privacy is dependent on the context from which the information will be collected as well as the kind of information being collected" (2013, p. 3). Addressing expectation, Alim proposes that "more research needs to be carried out regarding what social media users want and need in terms of ethics" (2014). At the center of this wide-ranging multidisciplinary ethics discussion lie the themes of context and expectation. These fundamental themes are equally central to ethics discussions in Library and Information Science.

\section{Ethics Discussion in Library and Information Science}

In the library profession, ethics discussions have traditionally centered around issues of patron privacy. Although Small, Kasianovitz, Blanford, and Celaya (2012) describe the library profession's understanding of privacy in networked communication as "ambiguous and evolving" 
(p. 175), they note that "patron privacy and confidentiality remain core values of libraries" (p. 187). The American Library Association's (ALA) Code of Ethics [i], Policy on Confidentiality of Library Records [ii], and Library Bill of Rights [iii] have been traditional frameworks for library ethics and privacy discussions in the United States, and International Federation of Library Associations (IFLA)'s Internet Manifesto 2014 [iv] states that it is a responsibility and role of library and information services to "strive to ensure the privacy of their users, and that the resources and services that they use remain confidential" (p. 2).

Library literature has often taken an absolutist view of patron privacy protection (Zimmer, 2013b). However, as early as the mid-1990s, some librarians began to question the traditional view of patron privacy. Datig and Russell (2014) note an ethical conflict between privacy and copyright, while Estabrook (1996) argues that "in the name of one good-keeping patron records confidential-we are sacrificing another: targeted and tailored services to library users" (p. 48). More recently, in response to "Web 2.0," the conversation has been revitalized, questioning how we can reconcile our ethical frameworks with the potential for patron data to inform and improve our library services (Zimmer, 2013a). Griffey (2010) ties this conversation specifically to SNSs, pointing out that the lines defining patron privacy become blurred for SNS usage in the library, since some patron information is being intentionally shared by the patrons themselves. Zimmer (2010) echoes the ethical discussion in other disciplines when he suggests that library users' expectations should be key ethical considerations, and that users may expect information shared on an SNS to be displayed and used only through that network. But even as libraries remain committed to the privacy of patron data, SNS data is released by patrons themselves into various public online environments.

Research using SNS data therefore lies in an ethical gray area, with idiosyncratic contexts defining the landscape. Users publish personal information online via SNSs, but do users perceive those SNSs to operate within a public space? Do users expect that the information posted will stay within the bounds of their own self-formed SNS community (Li et al., 2015)? Coeckelbergh (2011) notes that SNSs such as Twitter blur the distinction between public and private sphere, and Papathanassopoulos (2015) identifies broader shifts within the contemporary media landscape: "In this fragmented media milieu where the boundaries between offline and online, traditional and nontraditional media, personal life and public image are blurred, the very much talked-about-and difficult to conceptualize-issue of privacy is being readdressed" (p. 1). Indeed, the evolving practices of SNS users have given rise to new concepts to describe these new expectations. Twitter usage in particular illustrates an emerging and novel view towards communication in public spaces, that of the "personal public," where users' information exchange is characterized by personal relevance and conversational modes, and addressed to an audience comprised of explicit network connections (Schmidt, 2013, p. 4). The challenging topic of ethics in SNS research have been examined in other fields without consensus, as described in the literature review above. This ongoing discourse will benefit from libraries' unique perspective regarding patron privacy and information access. 
When examining SNS ethics in libraries, much of the existing literature focuses on patrons' use of SNSs while on library computers (Griffey, 2010) and educating patrons about privacy policies of SNSs (Gressel, 2014). In these scenarios, librarians are concerned about whether the information shared on an SNS is in fact patron data, and if so, how much responsibility falls on the library to safeguard the privacy of this data according to library ethical standards. Gressel (2014) argues that "the library has a larger responsibility to privacy, not only within the library, but [also] without" (p. 140). Taking on such responsibility over the ever-changing online realm may be a Sisyphean task, and this responsibility becomes even more complex as librarians turn to SNSs to conduct our own research. SNS-based research offers rich potential that could help us improve services and advance the field of Library Science. As with other disciplines, the data available from SNSs presents an opportunity for librarians to conduct user research on a large scale-an opportunity to understand our users in the aggregate in an unprecedented way.

Librarian-Researchers considering the ethics of SNS research will want to consider norms and expectations of the country where the SNS research is conducted. Governments may monitor and even censor SNS posts and legislative and library organization protections may not exist for personal privacy. In the United States in recent years, the ALA has bolstered its already-strong stance in support of personal privacy by releasing a resolution against the 2001 USA PATRIOT Act that characterizes sections of the Act as "a present danger to the ... privacy rights of library users" [viii], and by developing a Privacy Tool Kit [vi] that reinforces existing privacy documentation such as the Library Bill of Rights [ii]. The ALA's interpretation of the Library Bill of Rights affirms that "in a library (physical or virtual), the right to privacy is the right to open inquiry without having the subject of one's interest examined or scrutinized by others" [v]. In the case of SNS-based research, the open inquiry of a library user may in fact be subject to examination or scrutiny by others, including by librarians themselves who may be conducting research with user-generated SNS data. Another key-and possibly conflicting-ethos in libraries is that of information equality and openness of information. The ALA's interpretation of the Library Bill of Rights also affirms the library's "ethical imperative to provide unrestricted access to information and to guard against impediments to open inquiry." Libraries are proponents of Open Access, Open Data, and Linked Data-all ideas that embrace the free use of content on the Web. The nature of SNS data is unclear: is it patron data that should be tightly protected, or-because this data is often openly available on the Web-can it therefore be shared, reused, and remixed on a large scale in order to advance knowledge, scholarship, and library services? This fundamental question is highlighted through the examination of a case study of our own SNS-based research at the MSU Library.

\section{USE CASE: SNS RESEARCH}

In 2012, the MSU Library formed a Social Media Group. This group developed a Social Media Guide that established platform-specific goals, values, and other guidelines. To evaluate this initiative, we designed a research study to track changes in our Twitter community following the implementation of the Social Media Guide (Young and Rossmann, 2015). We used the Twitter API to build a dataset of publicly-available Twitter users and Twitter posts that we analyzed 
according to user type, post type, and interaction type. The dataset was comprised of user accounts and user activity from followers of the MSU Library Twitter account. We did not contact any Twitter users to seek consent to collect or analyze their data, nor has this research dataset been shared beyond the research team. With this study we aimed to improve our SNS efforts, and through the research we demonstrated that by following our Social Media Guide, the MSU Library's Twitter user community grew $100 \%$ in one year, with a corresponding increase of $275 \%$ in user interactions. The research results have informed additional refinement of the Social Media Guide, and have allowed us to improve our SNS programming, outreach, and services. On further reflection, however, our research design raised ethical concerns regarding the nature of library users, library records, and patron privacy.

\section{DISCUSSION}

\section{The Librarian-Researcher}

Researchers hold a standard of ethics regarding protecting the privacy, comfort, and safety of human research subjects. As van Wynsberghe et al. (2013) and Rivers and Lewis (2014) argue, privacy ethics are context-specific, and the library context is unique in its historical values. Librarians have traditionally held a further standard of ethics regarding the privacy of our library users. In our use case described above-and in similar research studies at other libraries (Kim, Abels, and Yang, 2012; Sewel, 2013; Shulman, Yep, and Tomé, 2015)—there emerges a dual role of Librarian-Researcher. We define Librarian-Researcher as a librarian who conducts his or her own research inquiries in the field of Library and Information Science. The role of Librarian-Researcher is distinct from other librarian roles, such as service provider, research assistant to patrons, and collaborator across the research spectrum. Like researchers in other disciplines, Librarian-Researchers formulate hypotheses, conduct research to support these hypotheses, and publish findings in order to advance knowledge in the field of Library Science. When we assume this dual role of Librarian-Researchers, we must operate within multiple contexts and recognize multiple ethical standards.

\section{SNS Data and Patron Data}

The library profession's history of protecting patrons and patron data suggests that SNS posts produced by a library's patrons may be subject to more rigorous protection than non-patron SNS data. The ALA Privacy Tool Kit recommends policy guidelines around protecting and preserving patron data and their Personally Identifiable Information (PII): "PII can easily be linked to every hash tag [sic], like, tweet, post, and social media interaction a user makes" [vii]. This definition and the Toolkit itself, provided by the library profession's oldest and largest organizing body, represent the contemporary expression of an historical ideal of privacy protection in libraries. By this definition, every SNS post and interaction produced by a library patron qualifies as a patron record and should therefore be protected as such. If the library profession adheres to its historical framework regarding patron privacy protection, these SNS interactions would be impractical and possibly ineligible for research. It may be undesirable, and even impossible, for Librarian-Researchers to continue to operate under such strict privacy standards. 
In 2013, Zimmer asked whether librarians should "loosen restrictions on collecting and retaining data to enhance ... services" (2013a, p. 51) . We assert that the answer to Zimmer's question is yes. Holding tight to an outdated system is detrimental to the growth of our library services. An inflexible view of patron privacy stands at odds not only with the nuanced ethical environment of social networks, but also with similarly long-standing library values surrounding information access. In essence, a tension exists between protecting and sharing SNS research data. In recent years, libraries have particularly championed open access and open data, embracing the data deluge as a fount of potentially transformative information. As stewards of information, including SNS user data, we aim to provide access to as wide an audience as possible, and to do so, it may be necessary to reevaluate our dogmatic view of patron privacy.

\section{SNS Research and Human Subject Research}

The SNS posts and interactions that we collected over the course of our research were produced by MSU Library users, and therefore raised questions about ethical data use. SNS research cannot simply be guided by the ethical standards that were designed for traditional, in-person research. Part of navigating the ethics of SNS research is to translate traditional ethical standards to online networked contexts, where data is plentiful and easy to collect passively without express permission from research subjects. But is studying SNS data equivalent to studying human subjects? Solberg (2010) argues that, although SNS data mining may qualify as human subject research, risks to the SNS users are sufficiently minor as to be exempt from federal human subject regulation. Wilkinson and Thelwall (2011) further argue that many SNS studies do not qualify as human subject research, suggesting that the objects of study are the text-based documents produced online by humans, and not the humans themselves. Neuhaus and Webmoor (2011), however, assert that SNS posts do in fact represent human subjects, and should be treated accordingly. If one concludes that studying SNS data is indeed human subject research, it raises the issue of informed consent, a discussion that has been especially prominent in the medical field (Vayena, Mastroianni, and Kahn, 2013; Chretien, 2013; Stephens, Williams, Gray, Kerby, and Wang, 2013).

The Association of Internet Researchers calls the concept of human subjects in SNS research "a persistent and contentious example of a dialectical tension between disciplinary/regulatory models and context-specific sensibilities," writing, "we encourage researchers to continue vigorous and critical discussion of the concept of 'human subject"' [viii, p. 6]. Indeed, our research would have been practically difficult and ethically questionable if we classified Tweets as human subjects, as this would have possibly violated two principles of the Librarian-Researcher: as librarians, the protection of personally identifiable information; as researchers, the safety, privacy, and informed consent of human subjects. The idea of tweets as texts moreover facilitates large-scale SNS research, and allows public sharing and reuse of research data.

\section{Research Data Sharing}

A hallmark of good research is reproducibility. Over the course of the research described in our case study, we collected tweets from 936 Twitter users. As Librarian-Researchers and open access advocates, we support data sharing, and we would like to share our dataset, both for reuse 
by other researchers, and in order to support the reproducibility of our results. Twitter's Terms and Conditions state the following: "If you provide Content to third parties, including downloadable datasets of Content or an API that returns Content, you will only distribute or allow download of Tweet IDs and/or User IDs" [x]. Within these terms, shared Twitter datasets such as ours may only include Tweet IDs, which reduces the associated metadata to a single field. These Tweet IDs can later be "hydrated" by a third party to display the full associated metadata, including such crucial fields as the Tweet text and the time and date of publication. However, deleted tweets or Twitter accounts that have been marked as private by the Twitter user will no longer be available and cannot be hydrated. It is important to note that in order for this practice to be considered ethical, it is necessary that tweets be classified as text, rather than human subjects, since hydrated tweets contain personally identifiable information.

By reducing our research dataset to Tweet IDs, we can share our data in compliance with Twitter's Terms and Conditions. However, a dataset of Tweet IDs is not a static object, and it has the potential to change over time. When a user deletes a Twitter post or sets his or her account as private, discrepancies can be introduced between the original dataset and the hydrated dataset. The research described in our case study is therefore likely unreproducible, as our SNS dataset exists as a snapshot in time that cannot be reconstituted retroactively by a third party. Given the dynamic nature of SNS data, completely reproducible SNS research-although still the ideal-may not be practical. SNS research may more appropriately be held to a standard of transparency. The question of research data sharing in the context of SNS data and PII is complicated and ongoing, and has yet further implications for ethical research practices. Tene and Polonetsky (2013) ask, "Could ethical scientific research be conducted without disclosing to the general public the data used to reach the results?" (p.256). In the first place, questions of ethics surround the sharing of sensitive research data. Secondly, questions of reproducibility surround the dynamic nature of SNS data. When taken together, SNS research data sharing is indeed shown to be quite problematic.

\section{ETHICAL FRAMEWORK}

\section{Design}

As of this article's publication, there is no existing ethical framework for conducting SNS research in libraries. This framework is informed primarily by the SNS ethics guidelines developed by van Wynsberghe et al. (2013), and by the Ethical Decision-Making and Internet Research Recommendations from the AoIR Ethics Working Committee [ix]. By surveying the literature in other disciplines, we have identified three key considerations that can inform our ethical practice in the field of Library Science: context, expectation, and value analysis. For each of these considerations, we have tailored the framework to consider ethical issues as they relate to libraries and our practice as Librarian-Researchers.

Ultimately, this framework aims to facilitate closer examination of research ethics, taking into account the details of each research project on a case-by-case basis. Ethical practice is rarely black-and-white. The proposed framework will benefit from the process-based approach 
described by AoIR [ix]. Librarian-Researchers should reevaluate the ethical implications of SNS data use at each stage of the research. The framework will also benefit from continuous internally-focused inquiry and externally-focused discussion over the course of a research project both within the library research team, and-if possible-with a trained ethicist.

\section{Context}

As discussed above, librarians in the United States bring a long history of valuing patron privacy protection into our research context. But our values are shifting from absolute patron privacy toward an ideology of open access and data sharing. Within the context of our own case study, we collected patron-generated Twitter data. Such SNS data could be considered less sensitive than more traditional forms of library patron data. Unlike circulation information or reference interaction data, which is "owned" by the library, Twitter data is hosted on a publicly-accessible platform to which its users have opted-in. Twitter's Privacy Policy details possible uses for what Twitter calls "non-private or non-personal information." The policy states that Twitter "may share or disclose your non-private, aggregated or otherwise non-personal information, such as your public user profile information, public Tweets, the people you follow or that follow you, or the number of users who clicked on a particular link" [xi]. However, when users opt-in to Twitter, it is unlikely that they expect to become the subjects of SNS research. Twitter's Terms and Conditions and Privacy Policy are several pages long and are updated frequently; the Terms and Conditions have been changed seven times since the site's launch in 2006 [xii]. An ethical evaluation of our use of SNS data in our case study was necessarily framed within context of that research. We also gained ethical insight by acknowledging that our research functions within the broader context of Twitter, where data sharing is both facilitated by an API and written into the Terms and Conditions.

\section{Expectation}

When conducting research, we must ask ourselves: What are our expectations as Librarian-Researchers, and what are the expectations of those we are researching? Each SNS platform constitutes a unique context that must be evaluated. The granularity of privacy mechanisms available to users on Facebook and Twitter, for example, vary widely (Damen and Zannone, 2013). Both SNS users and Librarian-Researchers may have different expectations from each platform. In our case study, we expected primarily to gain community insights by studying Twitter data. As Librarian-Researchers, we also expect to navigate the conflicted value system present within this context: ethical research standards, patron privacy, information access, and improved library services. The characteristics of SNSs challenge our notions of privacy, and can cause a turbulence that demands communication and conversation (Trepte, 2015). In a study of health care patients' use of SNSs, Antheunis (2013) found that $87.1 \%$ of survey respondents $(n=139)$ expected "that their privacy is guaranteed" (p. 429). Privacy on SNSs was seen as both a primary barrier to use and primary expectation of use for users in a healthcare context. Similar studies conducted across contexts and communities can help provide insight into the expectations of SNS users. In the case study presented here, we did not contact or study our community regarding their own expectations of SNS privacy and data reuse. In future studies, conducting interviews with or surveying SNS users could illuminate such expectations. 


\section{Value Analysis}

A value analysis evaluates whether the benefits of research conducted using SNS user data outweigh the possible risks to SNS users. Van Wynsberghe et al. (2013) recommend that a value analysis be conducted in partnership with an ethicist. In an academic library environment, ethicists can and should be tapped from other departments. While we did not involve an ethicist during the design planning of our research, a retroactive value analysis can be applied nonetheless. In our case, we collected SNS data that personally identified Library users. By analyzing Twitter accounts, Twitter posts, and Twitter interactions, we gained insight into the relationship between content and community (Young and Rossmann, 2015). We learned that our Twitter community favored certain types of content produced by the Library. We then configured our content creation on Twitter in response to the research results, which in turn generated additional community growth and increased interactions. The main value of this research is to improve our SNS services so that we are able to build an interested and interactive community. Our results have been shared within the library profession to aid the development of SNS services at other institutions.

On the one hand, we successfully employed SNS user data to improve SNS services and build community around library resources. On the other hand, we have exposed the privacy of our users by collecting and storing their data. Are the research results worth potential privacy violations? Have we acted ethically in using patron's tweets to inform community-building in our library and on campus? Such ethical questions are exceedingly difficult to answer. In our particular case, we have improved services to the advantage of our community, our library, and our profession. We propose that the benefit of improved services may outweigh the risks of privacy violation. With future research and increased complexity, we will seek to involve an ethicist to consult on these issues.

\section{Checklist}

Although Librarian-Researchers may not be ethicists, we must still confront complicated ethical issues when conducting our research. An itemized checklist necessarily condenses the complex components of an ethical analysis; this list is meant to guide Librarian-Researchers through a more in-depth analysis, in order to inform ethical SNS research design.

\section{Context}

$\square$ What are the SNS privacy norms in your culture or context?

$\square$ What are the laws, policies, and codes surrounding SNSs in your country, culture, library, and professional organization?

$\square$ What methods have you used to identify your study dataset?

$\square$ Are your data creators your own library users, or from a broader community?

$\square$ Which SNS platform(s) does your research analyze?

$\square$ What are the privacy policies and practices of the SNS platform(s)?

$\square$ How is user data made available by the SNS platform(s)? 
Expectation

What are your expectations as a Librarian-Researcher conducting your research?

$\square$ What are users' expectations of data reuse?

$\square$ What are the expectations of both the Librarian-Researcher and users specific to the SNS being studied?

$\square$ To what extent do users expect SNS data to be private?

Value analysis

$\square$ Consider your responses to the above questions regarding context and expectation.

$\square$ Consult with an ethicist, when appropriate and feasible.

$\square$ Determine the probability and magnitude of potential privacy risks.

$\square$ Determine the probability and magnitude of potential research gains.

$\square$ Measure the balance between risk and gain.

$\square$ If gain outweighs risk, proceed with research design.

$\square$ If risk outweighs gain, reevaluate and redesign research.

\section{CONCLUSION}

Librarian-Researchers are at a unique point in our history. In exploring SNSs as a source of data to conduct research and improve services, we become challenged by conflicting and equally cherished values of patron privacy and information access. By evaluating-and reevaluating-our research according to context, expectations, and value, we can provide ourselves with a path forward through a murky and seemingly irresolvable tension. This approach is limited, however, by the potentially cumbersome prospect of evaluating research on a case-by-case basis. In order to ethically reconcile the dual professional ideals of privacy and access, however, it may be necessary for Librarian-Researchers to integrate individual analyses into current and future SNS research studies. By embedding an ethical framework into practice, the Librarian-Researcher can help ensure that library SNS research aligns with both library values and research values, even as those values evolve with the changing data landscape.

\section{Acknowledgements}

The authors would like to thank the members of our Research and Writing Group for their feedback and support: Ryer Banta, Susan Borda, Kirsten Ostergaard, and Leila Sterman.

\section{REFERENCES}

[i] http://www.ala.org/advocacy/proethics/codeofethics/codeethics

[ii]

http://www.ala.org/advocacy/intfreedom/statementspols/otherpolicies/policyconfidentiality [iii] http://www.ala.org/advocacy/intfreedom/librarybill

[iv]

http://www.ifla.org/files/assets/faife/publications/policy-documents/internet-manifesto-2014. $\underline{\text { en.pdf }}$

[v] http://www.ala.org/advocacy/intfreedom/librarybill/interpretations/privacy 
[vi] http://www.ala.org/advocacy/privacyconfidentiality/toolkitsprivacy/privacy

[vii]

http://www.ala.org/advocacy/privacyconfidentiality/toolkitsprivacy/privacy-and-confidentialit y-library-core-values

[viii]

http://www.ala.org/Template.cfm?Section=ifresolutions\&Template=/ContentManagement/Cont entDisplay.cfm\&ContentID $=11891$

[ix] http://aoir.org/reports/ethics2.pdf

[x] https://dev.twitter.com/overview/terms/policy\#6. Be a Good Partner to Twitter

[xi] https://twitter.com/privacy?lang=en\#update

[xii] https://twitter.com/tos/previous?lang=en

Alim, S. (2014), "An initial exploration of ethical research practices regarding automated data extraction from online social media user profiles", First Monday, Vol. 19 No. 7.

Antheunis, M.L., Tates, K. and Nieboer, T.E. (2013), "Patients' and health professionals' use of social media in health care: Motives, barriers and expectations", Patient Education and Counseling, Vol. 92 No. 3, pp. 426-431.

Beaudoin, C.E. and Tao, C.-C. (2007), "Benefiting from social capital in online support groups: an empirical study of cancer patients.", Cyberpsychology \& behavior : the impact of the Internet, multimedia and virtual reality on behavior and society, Vol. 10 No. 4, pp. 587-90.

boyd, d. and Ellison, N.B. (2007), "Social Network Sites: Definition, History, and Scholarship", Journal of Computer-Mediated Communication, Vol. 13 No. 1, pp. 210-230.

Breiger, R.L. (2005), "Introduction to special issue: ethical dilemmas in social network research", Social Networks, Ethical Dilemmas in Social Network Research, Vol. 27 No. 2, pp. 89-93.

Burgess, J. and Bruns, A. (2012), “Twitter Archives and the Challenges of 'Big Social Data' for Media and Communication Research", M/C Journal, Vol. 15 No. 5, available at: http://www.journal.media-culture.org.au/index.php/mcjournal/article/view/561 (accessed 15 September 2014).

Chretien, K.C. (2013), "Social Media and Community Engagement in Trials Using Exception From Informed Consent", Circulation, Vol. 128 No. 3, pp. 206-208.

Coeckelbergh, M. (2011), "What are we doing?: Microblogging, the ordinary private, and the primacy of the present", Journal of Information, Communication and Ethics in Society, Vol. 9 No. 2, pp. 127-136. 
Damen, S. and Zannone, N. (2013), "Privacy Implications of Privacy Settings and Tagging in Facebook", in Jonker, W. and Petković, M. (Eds.),Secure Data Management, Lecture Notes in Computer Science, Springer International Publishing, pp. 121-138.

Datig, I. and Russell, B. (2014), "Instructing College Students on the Ethics of Information Use at the Reference Desk: A Guide and Literature Review", The Reference Librarian, Vol. 55 No. 3, pp. 234-246.

Dawson, P. (2014), "Our anonymous online research participants are not always anonymous: Is this a problem?”, British Journal of Educational Technology, Vol. 45 No. 3, pp. 428-437.

Elovici, Y., Fire, M., Herzberg, A. and Shulman, H. (2013), "Ethical Considerations when Employing Fake Identities in Online Social Networks for Research", Science and Engineering Ethics, Vol. 20 No. 4, pp. 1027-1043.

Estabrook, L.S. (1996), "Sacred trust or competitive opportunity: Using patron records", Library Journal, Vol. 121 No. 2, p. 48.

Gressel, M. (2014), “Are Libraries Doing Enough to Safeguard Their Patrons' Digital Privacy?”, The Serials Librarian, Vol. 67 No. 2, pp. 137-142.

Griffey, J. (2010), “Social Networking and the Library”, Library Technology Reports, Vol. 46 No. 8, pp. 34-37.

Gustafson, D.L. and Woodworth, C.F. (2014), "Methodological and ethical issues in research using social media: a metamethod of Human Papillomavirus vaccine studies", BMC Medical Research Methodology, Vol. 14 No. 1, p. 127.

Henderson, T., Hutton, L. and McNeilly, S. (2012), "Ethics and online social network research developing best practices", Proceedings of BCS HCI 2012 Workshops, pp. 1-4.

Hutton, L. and Henderson, T. (2013), “An Architecture for Ethical and Privacy-sensitive Social Network Experiments”, SIGMETRICS Perform. Eval. Rev., Vol. 40 No. 4, pp. 90-95.

Jansen, B.J., Zhang, M., Sobel, K. and Chowdury, A. (2009), "Twitter Power : Tweets as Electronic Word of Mouth", Journal of the American Society for Information Science and Technology, Vol. 60 No. 11, pp. 2169-2188.

Kaye, J. (2015), "The Tension Between Data Sharing and the Protection of Privacy in Genomics Research", in Mascalzoni, D. (Ed.), Ethics, Law and Governance of Biobanking, The International Library of Ethics, Law and Technology, Springer Netherlands, pp. 101-120. 
Kim, H.M., Abels, E.G. and Yang, C.C. (2012), "Who disseminates academic library information on Twitter?", Proceedings of the American Society for Information Science and Technology, Vol. 49 No. 1, pp. 1-4.

Langheinrich, M., Schmidt, A., Davies, N. and José, R. (2013), “A Practical Framework for Ethics: The PD-net Approach to Supporting Ethics Compliance in Public Display Studies", Proceedings of the 2Nd ACM International Symposium on Pervasive Displays, PerDis '13, ACM, New York, NY, USA, pp. 139-143.

Li, Y., Li, Y., Yan, Q. and Deng, R.H. (2015), "Privacy leakage analysis in online social networks", Computers \& Security, Vol. 49, pp. 239-254.

Neuhaus, F. and Webmoor, T. (2011), "Agile Ethics for Massified Research and Visualization”, Information, Communication \& Society, Vol. 15 No. 1, pp. 43-65.

Obole, A. and Welsh, K. (2012), "The danger of big data: Social media as computational social science", First Monday, Vol. 17 No. 7, doi:10.5210/fm.v17i7.3993.

Papathanassopoulos, S. (2015), “Privacy 2.0”, Social Media + Society, Vol. 1 No. 1.

Rinaldo, S.B., Tapp, S. and Laverie, D. a. (2011), “Learning by Tweeting: Using Twitter as a Pedagogical Tool”, Journal of Marketing Education, Vol. 33 No. 2, pp. 193-203.

Rivers, C.M. and Lewis, B.L. (2014), "Ethical research standards in a world of big data", F1000Research, doi:10.12688/f1000research.3-38.v2.

Schmidt, J. H. (2013). "Twitter and the rise of personal publics”, in Weller, K. et al. (Eds.), Twitter and Society. Peter Lang, New York, pp. 3-14.

Sewell, R.R. (2013), "Who is following us? Data mining a library's Twitter followers”, Library Hi Tech, Vol. 31 No. 1, pp. 160-170.

Shulman, J., Yep, J. and Tomé, D. (2015), “Leveraging the Power of a Twitter Network for Library Promotion”, The Journal of Academic Librarianship, Vol. 41 No. 2, pp. 178-185.

Small, H., Kasianovitz, K., Blanford, R. and Celaya, I. (2012), "What Your Tweets Tell Us About You: Identity, Ownership and Privacy of Twitter Data", International Journal of Digital Curation, Vol. 7 No. 1, pp. 174-197.

Solberg, L. (2010), "Data Mining on Facebook: A Free Space for Researchers or an IRB Nightmare?", University of Illinois Journal of Law, Vol. 2010 No. 2. 
Soranno, P.A., Cheruvelil, K.S., Elliott, K.C. and Montgomery, G.M. (2014), "It's Good to Share: Why Environmental Scientists' Ethics Are Out of Date”, BioScience, Vol. 65 No. 1, pp. 69-73.

Stephens, S.W., Williams, C., Gray, R., Kerby, J.D., and Wang, H. E. (2013), "Preliminary experience with social media for community consultation and public disclosure in exception from informed consent trials", Circulation, Vol. 128 No .3, pp. 267-270.

Tene, O. and Polonetsky, J. (2013), "Big Data for All: Privacy and User Control in the Age of Analytics", Northwestern Journal of Technology and Intellectual Property, Vol. 11 No. 5, pp. 239-273.

Trepte, S. (2015), "Social Media, Privacy, and Self-Disclosure: The Turbulence Caused by Social Media's Affordances”, Social Media + Society, Vol. 1 No. 1.

Tumasjan, A., Sprenger, T.O., Sandner, P.G. and Welpe, I.M. (2010), "Predicting Elections with Twitter: What 140 Characters Reveal about Political Sentiment.”, ICWSM, Vol. 10, pp. 178-185.

Van Wynsberghe, A.L., Been, H. and van Keulen, M. (2013), To use or not to use: guidelines for researchers using data from online social networking sites, available at:

http://purl.utwente.nl/publications/87936 (accessed 20 January 2015).

Vayena, E., Mastroianni, A.C., and Kahn, J.P. (2013), "Caught in the Web: Informed Consent for Online Health Research.” Science Translational Medicine, Vol. 5 No. 173), pp. 1-3.

Wilkinson, D. and Thelwall, M. (2011), "Researching Personal Information on the Public Web: Methods and Ethics", Social Science Computer Review, Vol. 29 No. 4, pp. 387-401.

Young, S.W.H. and Rossmann, D. (2015), "Building Library Community Through Social Media”, Information Technology and Libraries, Vol. 34 No. 1, pp. 20-37.

Zimmer, M. (2010), “'But the data is already public': on the ethics of research in Facebook”, Ethics and Information Technology, Vol. 12 No. 4, pp. 313-325.

Zimmer, M. (2013a), “Patron Privacy in the '2.0' Era”, Journal of Information Ethics, Vol. 22 No. 1, pp. 44-59.

Zimmer, M. (2013b), “Assessing the Treatment of Patron Privacy in Library 2.0 Literature”, Information Technology and Libraries, Vol. 32 No. 2, pp. 29-41. 
